\title{
Cambio climático y salud: retos para Colombia
}

\author{
Climate change and health: challengues for Colombia
}

Daniel Elias Cuartas ${ }^{1}$, Fabián Méndez ${ }^{1}$

Forma de citar: Cuartas DE, Méndez F. Cambio climático y salud: retos para Colombia. Rev Univ Ind Santander Salud. 2016; 48(4):428-435. DOI: http://dx.doi.org/10.18273/revsal.v48n4-2016001 (c) (1)

\section{RESUMEN}

El cambio climático impactará múltiples ámbitos de la sociedad, incluyendo la salud. Sus efectos en salud se deberán al aumento de la temperatura y al incremento/disminución de la precipitación y en especial al incremento de la frecuencia y magnitud de eventos climáticos extremos. El incremento de la morbilidad y mortalidad en salud se presentará a través de diversos mecanismos imbricados con elementos de los sistemas sociales y naturales de los socio - ecosistemas. La respuesta a dicha complejidad requiere abordajes y perspectivas interdisciplinarias que evalúen la vulnerabilidad de las poblaciones sensibles y que se piensen estrategias de adaptación adecuadas a los contextos locales. En este sentido el presente documento se propone describir los posibles efectos del cambio climático en la salud y plantea los principales retos para el estudio de dicha relación en el contexto Colombiano.

Palabras claves: Cambio climático, salud, temperatura.

\begin{abstract}
Climate change will affect multiple areas of society, including health. Its health effects will be due to rising temperatures and increased / decreased precipitation and especially the increased frequency and magnitude of extreme weather events. Increased morbidity and mortality in health will be presented through various intertwined with elements of social and natural systems of socio mechanisms - ecosystems. The answer to this complexity requires interdisciplinary approaches and perspectives to assess the vulnerability of sensitive populations and adaptation strategies appropriate to local contexts think. In this sense, this document is to describe the possible effects of climate change on health and pose major challenges for the study of the relationship in the Colombian context.
\end{abstract}

Keywords: Climate change, health, temperature.

1. Universidad del Valle. Cali, Colombia.

Correspondencia: Daniel Elías Cuartas. Dirección: Universidad del Valle, Calle 4B \# 36-140 Edificio 118, Oficina 215. Correo electrónico: daniel.cuartas@correounivalle.edu.co. Teléfono +52 3163268449. 


\section{INTRODUCCIÓN}

El sistema climático ha cambiado repetidamente en los 4600 millones de años de la historia de la tierra, alternándose glaciaciones y clima cálido, como resultado de cambios en la distribución de la radiación solar y de la composición de la atmosfera, debido a variaciones en la excentricidad de la órbita de la tierra alrededor del sol, en el grado de inclinación de la tierra respecto al plano de la órbita y en el movimiento de precesión que realiza la tierra respecto a su eje de rotación ${ }^{1-3}$. Otros factores vinculados con los cambios en el clima de la tierra han sido las fluctuaciones solares, la actividad volcánica, el vapor de agua y la concentración de gases efecto invernadero ${ }^{4}$.

La última glaciación que tuvo lugar en la historia geológica de la tierra ocurrió hace aproximadamente unos 100.000 años y finalizó hace 12.000 años, tiempo de inicio de la época reciente conocida como holoceno, en el cual la temperatura promedio aumento cerca $5^{\circ} \mathrm{C}$ y el deshielo incrementó el nivel del mar e inundó grandes extensiones de la tierra ${ }^{4-6}$.

El cambio climático actual se encuentra ampliamente relacionado con la actividad humana, sus impactos se reflejan a nivel global, especialmente en regiones con ecosistemas vulnerables sensibles al clima ${ }^{7}$. Los impactos del cambio climático pueden afectar de diferente manera el desarrollo humano, en cinco ámbitos de la vida, de acuerdo con el PNUD (2007): el primero es el impacto en la producción agrícola y la seguridad alimentaria, el segundo es el estrés por falta de agua e inseguridad del agua, el tercero es el aumento en el nivel del mar y la exposición a desastres meteorológicos, el cuarto es la transformación de los ecosistemas y la disminución de la biodiversidad, el quinto son los impactos en la salud humana ${ }^{4}$.

En este contexto y de acuerdo con el IPCC, hay pruebas suficientes de que el cambio y la variabilidad climática, actualmente contribuyen a la carga mundial de enfermedad y muerte prematura y que dichos efectos se incrementarán en los próximos $a_{n ̃ o s}^{8}$. Los impactos más importantes del cambio climático pueden deberse a cambios en los eventos extremos ${ }^{9}$, en enfermedades infecciosas, lesiones, cáncer y golpes de calor entre otras por lo que este documento se propone describir los posibles efectos del cambio climático en la salud y plantear los principales retos para el estudio de dicha relación en el contexto Colombiano.

\section{Sistema Climático de la tierra}

El sistema climático de la tierra es el resultado de una compleja interacción entre la radiación solar, la hidrosfera, la atmosfera, la criosfera, la litosfera, la biosfera, los océanos y los fenómenos climáticos emergentes ${ }^{10}$. La radiación solar es la fuente de energía que influye en el sistema climático de la tierra, del total de la radiación emitida por el sol, el $8 \%$ corresponde con ultravioleta, la cual es reflejada en un $30 \%$ por las nubes, las moléculas del aire, el polvo la neblina y la superficie de la tierra, un $23 \%$ es absorbida por el ozono en la atmosfera, las nubes y el vapor de agua y el $47 \%$ restante se absorbe como radiación directa o difusa. La tierra también emite radiación pero mucha de esta es reabsorbida por medio del efecto invernadero y mantiene las condiciones calientes que facilitan la vida ${ }^{11}$.

La dinámica es una de las principales características del clima y está determinada por los procesos de circulación atmosférica debidos a que el ecuador recibe más radiación que los polos ${ }^{12} \mathrm{y}$ a la circulación del agua oceánica determinada por la temperatura del agua y por la salinidad ${ }^{10}$. Así mismo, la geomorfología afecta la circulación atmosférica local y la climatología por medio de efectos orográficos, debidos a la presencia de montañas ${ }^{12,13}$ y la vegetación influye en el clima por medio de la eficiencia del intercambio de agua y energía ${ }^{14}$.

\section{Eventos Climáticos Extremos}

Un evento climático extremo es la ocurrencia de un evento de alguna variable climática (precipitación, temperatura, humedad relativa) con un valor por encima o por debajo de los valores altos o bajos del rango de observación histórico de esa variable ${ }^{15}$. Los eventos climáticos extremos pueden clasificarse como simples, valores altos o bajos de temperatura y precipitación y complejos como inundaciones, sequías y tormentas ${ }^{16}$.

\section{Variabilidad Climática}

La variabilidad climática se refiere a la variación de los parámetros del clima respecto el promedio del clima (resultado de una observación por un largo periodo de tiempo), en una región específica ${ }^{17}$. La variabilidad climática puede ser interanual en la que se observa como es el comportamiento del clima en un año, 5 o hasta 10 años, respecto al promedio histórico dada una serie de mínimo 30 años. Puede estar asociada con cambios en la atmosfera y en el océano, como es el caso de fenómeno de oscilación sur - ENSO, también conocido como 
El Niño/Niña ${ }^{18}$ o puede ser intra-anual debida a las variaciones diarias o estacionales de la radiación solar. Se observa en escala de estaciones, meses, semanas o días que resultaron más lluviosas o secos, más calientes o fríos respecto al periodo histórico.

\section{Cambio climático}

En el primer reporte del IPCC publicado en 1990 se planteó la certeza que en el sistema tierra hay un efecto invernadero de origen natural que mantiene la tierra caliente y que las actividades humanas generan emisiones de estos gases efectos invernadero como dióxido de carbono, metano, clorofluorocarbonados y óxido nitroso, con el potencial de incrementar el efecto invernadero, lo que resultaría en un incremento de la temperatura ${ }^{19}$.

En el último informe de 2014, el IPCC plantea que sin lugar a dudas el sistema climático se está calentando desde 1950 y que es clara la influencia humana debido a que las emisiones de gases de efecto invernadero de los últimos siglos han producido un forzamiento radiactivo positivo (para el 2011 fue mayor en un 43\% respecto el 2005), lo que se ha traducido en un aumento de la absorción de energía en el sistema climático y se evidencia en el aumento de las temperaturas ${ }^{20,21}$.

En la temperatura de la atmósfera se ha detectado una tendencia de calentamiento de $0.85^{\circ} \mathrm{C}$ en promedio entre 1880-2012, y en los océanos se ha acumulado el $90 \%$ de la energía incrementada entre 1971 y 2010. De igual forma existe certeza que el nivel del mar se ha incrementado $1.7 \mathrm{~mm}$ entre 1900-2010, también se ha aumentado el carbono antropogénico y está disminuyendo la extensión de los mantos de hielo y los glaciares ${ }^{22}$.

También se ha observado cambios en los eventos climáticos extremos, disminución de las noches y días fríos y un aumento de las noches y días cálidos, aumento de las olas de calor en Europa, Asia y Australia. Al igual existe la probabilidad que en algunas zonas del planeta las precipitaciones intensas aumenten, mientras en otras áreas disminuyan. En términos de eventos extremos con un nivel de confianza medio se espera para Suramérica un incremento de los días y noches cálidas y una disminución de los días y las noches frías; en tanto se espera un incremento de eventos de precipitación extremas con variaciones espaciales ${ }^{23}$.

De acuerdo con las proyecciones se espera que la temperatura media global aumente entre $0.3-0.7^{\circ} \mathrm{C}$ entre 2016-2035 respecto 1986-2005. Es altamente probable que se incrementen el número de temperaturas extremas calientes y se presenten más frecuentemente olas de calor. Respecto a la precipitación global existe un alto nivel de certeza que se incremente los niveles de precipitación media y los eventos extremos, en la medida que vaya aumentando la temperatura en las regiones del mundo ${ }^{24}$.

A nivel nacional la tercera comunicación de cambio climático del 2015 presenta un escenario para Colombia caracterizado por un aumento en la temperatura que podría ser de $2.14^{\circ} \mathrm{C}$ para 2100 , relacionado con el consecuente incremento del nivel del mar, la modificación de la línea de costa, el derretimiento de los picos nevados y la retracción del sistema de páramos en Colombia. En tanto a las precipitaciones se espera una reducción entre el 10-30\% en departamentos como Amazonas, Vaupés, sur del Caquetá, San Andrés y Providencia, Bolívar, Magdalena, Sucre y norte del Cesar, mientras tanto en otros departamentos se espera un incremento en igual rango en Nariño, Cauca, Huila, Tolima, eje cafetero, occidente de Antioquia, norte de Cundinamarca, Bogotá y centro de Boyacá ${ }^{25}$.

\section{Cambio climático y salud}

Existe una amplia variedad de efectos en salud debidos al cambio climático que se presentan actualmente y se presentarán en el tiempo. Comprender la manera en que estos suceden supone un reto debido a que los efectos serán diferenciales en tiempo y en espacio. Son requeridos, por lo tanto, abordajes sistémicos y complejos para entender las múltiples relaciones que se presentan, de forma que se puedan desarrollar medidas de adaptación adecuadas a los contextos, políticos, económicos, ecológicos, sociales y ambientales.

El cambio climático es incontrovertible, evidenciado en los actuales y futuros incrementos de la temperatura, del $\mathrm{CO}^{2}$, del nivel del mar, y de la frecuencia y magnitud de eventos climáticos extremos, así como aumentos o reducciones del nivel de precipitaciones, condiciones cambiantes que afectan los patrones de enfermedad y la carga de morbilidad y mortalidad. Estos efectos están mediados por las condiciones y la capacidad de respuesta de cada sociedad, determinando un nivel de vulnerabilidad que es necesario conocer para el desarrollo de estrategias de adaptación.

Diferentes mecanismos pueden darse para que el cambio climático produzca efectos en la salud ${ }^{26}$. En primer lugar el estrés climático ya sea por altas y bajas temperaturas y precipitaciones que resultan en inundaciones, tormentas, sequias, incendios, disminución en la producción de alimentos, pueden aumentar la carga de morbilidad y mortalidad por lesiones y nutrición. Un segundo 
mecanismo se presenta a través de la proliferación de microorganismos bacterianos y virus que contaminan los alimentos y el agua.

Un tercer mecanismo se presenta en los cambios en la ecología de los vectores y en la relación vector-patógeno - hospedero, la cual puede sufrir modificaciones temporales y espaciales, dado que se puede ampliar o reducir el área en las cuales hay presencia del vector, sufrir cambios estacionales o acelerar el ciclo de vida de los vectores o el ciclo de reproducción de los patógenos.
El cuarto mecanismo se puede presentar al afectar las cosechas, la producción pecuaria y piscícola, con efectos en el acceso de alimentos, la nutrición y la salud. Finalmente el quinto mecanismo se presenta como efectos de la perdida de enseres, bienes y en algunos casos desplazamiento como resultado de eventos climáticos extremos, como inundaciones, sequías, derrumbes, que aumentan la pobreza, afecta la salud mental, incrementa la posibilidad de enfermedades infecciosas, la malnutrición y la exposición a riesgos físicos, (Figura 1).

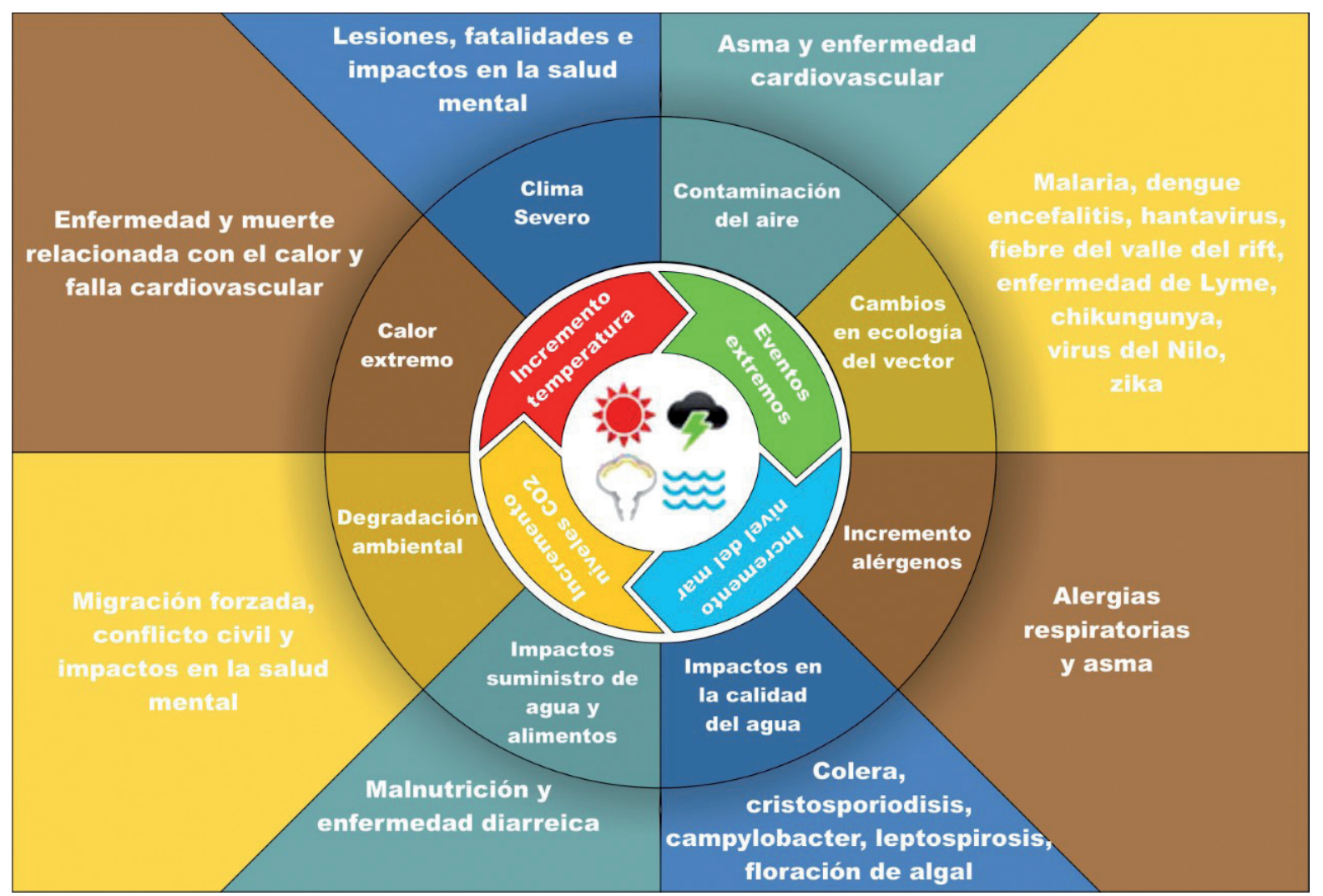

Figura 1. Impactos del cambio climático en salud Fuente: George Luber, Climate and Health Program, CDC

Desde esta perspectiva, la interacción entre los eventos climáticos y la morbilidad y mortalidad en salud no es completamente directa y lineal, existen factores ambientales, sociales, físicos, químicos, biológicos, económicos e incluso de la geografía local que actúan como moderadores de los efectos en salud que se presentan o presentarán ${ }^{27,28}$.

En algunos casos puede encontrarse una direccionalidad, como es el caso de la morbilidad y mortalidad por cáncer de piel y por golpes de calor los cuales están fuertemente asociados con la exposición a la radiación ultravioleta y olas de calor de respectivamente. Aunque podrían considerarse algunos factores mediadores, como la condición física, estos efectos tienden a tener una relación directa con el evento climático.

Por otro lado, es posible reconocer dos tipos de moderadores de los efectos del cambio climático en la salud. Por un lado se presentan aquellos relacionados con sistemas naturales, como factores biológicos, físicos, químicos y ecológicos y otros relacionados con sistemas humanos en los que se encuentran factores sociales, culturales, económicos, políticos, de infraestructura, institucionales, comunitarios e históricos ${ }^{29,30}$, (Tabla 1). 
Tabla 1. Efectos en la salud relacionados con cambio climático

\begin{tabular}{|c|c|c|c|}
\hline Evento o variable climática & Impacto en el ambiente & Moderadores del efecto & Evento en salud \\
\hline \multirow{7}{*}{$\begin{array}{l}\text { Cambios en los promedios de } \\
\text { temperatura y precipitación y } \\
\text { eventos climáticos extremos }\end{array}$} & Olas de calor & Directa & $\begin{array}{l}\text { Cardiovascular } \\
\text { Enfermedad asociada al calor }\end{array}$ \\
\hline & Degradación del ozono & Directa & $\begin{array}{c}\text { Cáncer de piel, lesiones } \\
\text { piel, lesiones oculares }\end{array}$ \\
\hline & $\begin{array}{l}\text { Inundaciones, sequías y } \\
\text { tormentas }\end{array}$ & $\begin{array}{c}\text { Directa } \\
\text { Sistemas humanos }\end{array}$ & Lesiones, fatalidades \\
\hline & $\begin{array}{l}\text { Contaminación del aire e } \\
\text { incremento de alérgenos }\end{array}$ & $\begin{array}{l}\text { Directa } \\
\text { Sistemas naturales } \\
\text { / humanos }\end{array}$ & Alergias respiratorias y asma \\
\hline & $\begin{array}{l}\text { Clima intenso y degradación } \\
\text { ambiental }\end{array}$ & Sistemas humanos & $\begin{array}{l}\text { Salud mental } \\
\text { Migración }\end{array}$ \\
\hline & $\begin{array}{l}\text { Contaminación del agua, } \\
\text { sequías, inundaciones }\end{array}$ & $\begin{array}{l}\text { Sistemas naturales / } \\
\text { humanos }\end{array}$ & $\begin{array}{c}\text { Enfermedades asociadas al } \\
\text { agua, diarrea, infecciosas, } \\
\text { malnutrición }\end{array}$ \\
\hline & $\begin{array}{l}\text { Ecología del vector, virus } \\
\text { y parásitos, pérdida de } \\
\text { biodiversidad }\end{array}$ & $\begin{array}{l}\text { Sistemas naturales } \\
\text { / humanos }\end{array}$ & Enfermedades infecciosas \\
\hline
\end{tabular}

Fuente: Basado en Ahern \& McMichael (2002), Confalonieri et al. (2007), Frumkin, Hess, Luber, Malilay, \& McGeehin (2008), Haines et al. (2006), IPCC (2014), McMichael et al. (2006), OPS (2008), Organización Panamericana de la Salud - OPS (2011b), J. Patz et al. (2008), Portier CJ (2010), WHO. \& WMO. (2012).

El reconocer este par de sistemas no significa una escisión hombre - naturaleza, por el contrario permite identificar y clasificar los elementos de un socio ecosistema, con el objetivo de reconocer relaciones, interacciones y realimentaciones, para una comprensión sistémica, donde la sensibilidad a las condiciones iniciales y las propiedades emergentes sean posibles.

En este sentido, los efectos del cambio climático en las enfermedades infecciosas están altamente condicionados por los impactos que pueda tener sobre el ciclo de vida y de transmisión de los agentes infecciosos, los cuales pueden llegar a ser atenuados y por lo tanto irreconocibles, debido al control vectorial, los cambios en la infraestructura, el desarrollo de estrategias de educación entre otros ${ }^{31,32}$. Por su parte los eventos climáticos extremos tienen el potencial de causar los mayores efectos debido a su condición de ser eventos agudos que se presentan en el corto plazo $^{33,34}$.

Para las enfermedades respiratorias, las condiciones climáticas influencia la calidad del aire determinada a través de la modificación de las concentraciones de contaminantes en la atmosfera. Se espera que las concentraciones del ozono aumenten en algunas regiones del planeta, lo que impactará la morbilidad y mortalidad debido a que este gas está asociado con la reducción de la función pulmonar, la exacerbación de enfermedades respiratorias crónicas y el incremento de las admisiones en hospitales. Por su parte no existe claridad sobre como el cambio climático afectará las concentraciones de material particulado que tienen efectos similares al del ozono ${ }^{35,36}$.

En el caso de las enfermedades asociadas al agua, las precipitaciones extremas tienen el potencial de generar descargas de contaminantes en los acuíferos, como efectos del lavado que sufre la superficie del suelo. Al tener cuencas hidrográficas altamente intervenidas con procesos intensivos de agricultura y de producción pecuaria y fuertes procesos erosivos, al momento de ocurrir una precipitación extrema se produce un lavado del suelo que arrastra sedimentos, pesticidas, insecticidas, materia orgánica, que termina en los ríos que abastecen de agua potable las poblaciones. Estas descargas generan procesos de enfermedad o en otros casos suspensión del servicio de agua potable, debido a la incapacidad de las plantas de potabilización para tratar este tipo de aguas ${ }^{37-39}$.

Los efectos de cambio climático en la salud son múltiples, complejos y dependientes de la naturaleza de cada enfermedad. No es posible abordar el impacto del cambio climático de forma lineal, se requieren abordajes complejos que aborden múltiples factores y esto supone retos y desafíos para cada sociedad, en especial para los países en vías de desarrollo, que aportan poco en el total de gases efectos invernadero y verán afectados en gran medidas sus socio - ecosistemas. 
Retos para el estudio de la relación cambio climático y salud en Colombia

El cambio climático favorecerá o disminuirá la carga de morbilidad y mortalidad en salud, por lo que se requiere incrementar la compresión como esto sucederá31. En Colombia se producirán impactos diferenciales, según las características de las regiones del país, dadas las proyecciones del aumento de la temperatura, incrementos/disminución de la precipitación y el incremento de la frecuencia y magnitud de los eventos climáticos extremos ${ }^{8}$.

Un aspecto relevante para el contexto colombiano es la identificación del nivel de vulnerabilidad nacional y local, así como la identificación de los riesgos claves ${ }^{40}$. La vulnerabilidad entendida como una función de la exposición a las condiciones climáticas asociadas al cambio climático, la sensibilidad o respuesta de los socio - ecosistemas a determinada exposición y la capacidad de adaptación reconocible a partir de las capacidades, sociales, institucionales y recursos con que se cuenten ${ }^{41,42}$.

Sin embargo, para poder conocer la vulnerabilidad se requiere contar y acceder a información y datos de indicadores de variables climáticas, sociales, institucionales, a diferentes escalas, municipales, departamentales y nacionales. Respecto a las variables climáticas se debe hacer un reconocimiento de los indicadores de eventos climáticos extremos que se relacionan con el evento en salud de interés y reconocer la escala temporal de la enfermedad para calcular los eventos climáticos extremos en un tiempo similar ${ }^{43}$.

Para lo anterior se requiere incrementar la vigilancia en salud, mejorar los sistemas de predicción, desarrollar sistemas de monitorio y observatorios que adquieran y procesen la información, con el objetivo de conocer el nivel de vulnerabilidad y esta información sea la base para la toma de decisiones ${ }^{33}$. Una ventaja en este sentido es la Ley 1712 de 2014 que regula el derecho al acceso a la información pública nacional, bajo la cual se regula la forma en que se puede obtener gratuitamente la información de las instituciones públicas.

Sin embargo, es necesario que la academia se articule con las instituciones y la comunidad, con el objetivo de construir estrategias de adaptación que permitan incrementar la compresión de los efectos en salud del cambio climático por medio de aproximaciones efectivas para la prevención y reducción de la vulnerabilidad, especialmente en poblaciones altamente sensibles, así como la evaluación de los impactos en salud de las políticas para el cambio climático.

En este sentido, Colombia debe iniciar un proceso articulado de adaptación al cambio climático que desborde el área de la salud, porque la salud es el resultado interacciones complejas en la sociedad ${ }^{44}$ y los impactos no podrán ser abordados, mitigados y reducidos desde posturas individuales, disciplinares e institucionales, por el contrario, perspectivas interdisciplinarias sustentadas en el reconocimiento de otros tipos de conocimientos, son las que deben guiar el análisis de vulnerabilidad y la construcción de estrategias de adaptación.

\section{AGRADECIMIENTOS}

A Colciencias por administrar los recursos de los colombianos que financian los estudios doctorales de Daniel Elías Cuartas y al International Development Research Centre - IDRC de Canadá, por la financiación del proyecto "Cambio Climático, Vulnerabilidad y Salud en Colombia".

Nota: los desarrollos conceptuales presentados en este documento son resultado del trabajo realizado en el marco del proyecto de investigación "Cambio Climático, Vulnerabilidad y Salud en Colombia" financiado por el International Development Research Centre - IDRC de Canada

\section{REFERENCIAS}

1. Intergovernmental Panel on Clima Change - IPCC. Climate Change 2007: The Physical Science Basis. United Kingdom and New York: 2007.

2. Petit JR, Jouzel J, Raynaud D, Barkov NI, Barnola $\mathrm{JM}$, Basile I, et al. Climate and atmospheric history of the past 420,000 years from the Vostok ice core, Antarctica. Nature. 1999; 399: 429-436. DOI: $10.1038 / 20859$.

3. Tzedakis PC, Andieu V, Beaulieu J-ld, Crowhurst S, Follieri M, Hooghiemstra H, et al. Comparison of terrestrial and marine records of changing climate of the last 500000 years. Earth Planet Sci Letters. 1997; 150: 171-176. DOI: 10.1016/S0012821X(97)00078-2.

4. PNUD. Informe sobre desarrollo humano 2007 2008. La lucha cotra el cambio climático: solidaridad frente a un mundo dividido. New York Programa de las Naciones Unidas para el Desarrollo; 2007.

5. Juan torres, Gómez A. Adaptación al cambio climático: de los fríos y los calores en los Andes. 
Lima: Soluciones Prácticas - ITDG, 2008.

6. Mayewski PA, Rohling EE, Stager JC, Karle'n W, Maasch KA, Meeker LD, et al. Holocene climate variability. Quaternary Research. 2004; 62(3): 243255. DOI:10.1016/j.yqres.2004.07.001.

7. Bailey C, Convery I, Mort M, Baxter J. Different public health geographies of the 2001 foot and mouth disease epidemic: 'citizen' versus 'professional' epidemiology. Health Place. 2006; 12(2): 157-166. DOI: 10.1016/j.healthplace.2004.11.004.

8. Confalonieri U, Menne B, Akhtar R, Ebi KL, Hauengue M, Kovats RS, et al. Human health. Climate Change 2007: Impacts, Adaptation and Vulnerability. Contribution of Working Group II to the Fourth Assessment Report of the Intergovernmental Panel on Climate Change. Cambridge, UK: 2007.

9. Guttman NB. Statistical descriptors of climate. Bull Am Meteor Society. 1989; 70(6): 602607. DOI: http://dx.doi.org/10.1175/15200477(1989)070<0602:SDOC $>2.0$. CO;2

10. Cronin TM. Paleoclimates: understanding climate change past and present. Press $\mathrm{CU}$, editor. New York: Columbia University Press; 2010.

11. Trenberth KE, Fasullo JT, Kiehl J. Earth's Global Energy Budget. Bull Am Meteor Society. 2009; 90(3): 311-323. DOI: http://dx.doi. org/10.1175/2008BAMS2634.1.

12. Chapin III ES, Matson PA, Vitousek PM. Principles of Terrestrial Ecosystem Ecology. New York: Springer New York; 2012.

13. Eslava JA. Climatología y diversidad climática de Colombia. Rev Acad Colomb Ciencias. 1993; 18(71): 507-538.

14. Bureau of Meteorology. The greenhouse effect and climate change. Australia: 2003.

15. IPCC. Managing the Risks of Extreme Events and Disasters to Advance Climate Change Adaptation. A Special Report of Working Groups I and II of the Intergovernmental Panel on Climate Change. Cambridge, UK: 2012.

16. WORLD HEALTH ORGANIZATION - WHO. Climate change and human health risks and responses. Geneva: 2003.

17. Food and Agriculture Organization of The United Natios - FAO. Climate variability and change: adaptation to drought in Bangladesh A resource book and training guide Rome 2007.

18. Fedorov AV, Philander SG. Is El Niño changing? Science. 2000; 288(5473): 1997-2002.

19. Intergovernmental Panel on Clima Change - IPCC. Climate Change the IPCC Scientific Assessment. New York: 1990.
20. IPCC. Climate Change 2013: The Physical Science Basis. Contribution of Working Group I to the Fifth Assessment Report of the Intergovernmental Panel on Climate Change. Cambridge, United Kingdom and New York, NY, USA: 2013.

21. Stocker TF, Qin D, Plattner GK, Alexander N, Tignor M, Allen SK, et al. Resumen técnico. En: Cambio climático 2013. Bases físicas. Contribución del Grupo de trabajo I al Quinto Informe de Evaluación del Grupo Intergubernamental de Expertos sobre el Cambio Climático. Reino Unido y Nueva York, NY, Estados Unidos de América: 2013. DOI: 10.1017/ CBO9781107415324.

22. Rhein M, Rintoul SR, Aoki S, Campos E, Chambers D, Feely RA, et al. Observations: Ocean. In: Stocker TF, Qin D, Plattner GK, Tignor M, Allen SK, Boschung J, et al. Climate Change 2013: The Physical Science Basis contribution of Working Group I to the Fifth Assessment Report of the Intergovernmental Panel on Climate Change. United Kingdom and New York, NY, USA: Cambridge University Press, Cambridge; 2013.

23. Hartmann DL, Tank MGK, Rusticucci M, Alexander LV, Brönnimann S, Charabi Y, et al. Observations: atmosphere and surface. In: Stocker TF, D. Qin, G.K. Plattner, M. Tignor, S.K. Allen, J. Boschung, A. Nauels, Y. Xia, V. Bex and P.M. Midgley, editor. Climate Change 2013: The Physical Science Basis Contribution of Working Group I to the Fifth Assessment Report of the Intergovernmental Panel on Climate Change. United Kingdom and New York, NY, USA: Cambridge University Press, Cambridge; 2013.

24. Collins MR, Knutti J, Arblaster JL, Dufresne T, Fichefet P, Friedlingstein X, Gao WJ, Gutowski T. Long-term Climate Change: Projections, Commitments and Irreversibility. In: Stocker T.F. DQ, G.-K. Plattner, M. Tignor, S.K. Allen, J. Boschung, A. Nauels, Y. Xia, V. Bex, P.M. Midgley, editor. Climate Change 2013: The Physical Science Basis Contribution of Working Group I to the Fifth Assessment Report of the Intergovernmental Panel on Climate Change Cambridge, United Kingdom and New York, NY, USA: Cambridge University Press; 2013.

25. IDEAM P, MADS, DNP, CANCILLERÍA. Nuevos Escenarios de Cambio Climático para Colombia 2011-2100 Herramientas Científicas para la Toma de Decisiones - Enfoque Nacional - Departamental: Tercera Comunicación Nacional de Cambio Climático. Bogotá: 2015.

26. Luber G. Impact of climate change on human helath. Atlanta, EEUU: CDC; 2014. 
27. Haines A, Kovats RS, Campbell-Lendrum D, Corvalan C. Climate change and human health: impacts, vulnerability and public health. Public Health. 2006; 120(7): 585-596. DOI: 10.1016/j. puhe.2006.01.002.

28. OPS OPdlS-. Cambio climático y salud humana: riesgos y respuestas_ resumen actualizado. Washington: 2008.

29. Portier CJ, Thigpen Tart K, Carter SR, Dilworth $\mathrm{CH}$, Grambsch AE, Gohlke J, et al. A Human Health Perspective On Climate Change: A Report Outlining the Research Needs on the Human Health Effects of Climate Change. 2010. DOI:10.1289/ehp.1002272.

30. Patz J, Campbell Lendrum D, Gibbs H, Woodruff R. Health impact assessment of global climate change: expanding on comparative risk assessment approaches for policy making. Annu Rev Public Health. 2008; 29: 27-39. DOI: 10.1146/annurev. publhealth.29.020907.090750.

31. Altizer S, Ostfeld RS, Johnson PTJ, Kutz S, Harvell CD. Climate Change and Infectious Diseases: From Evidence to a Predictive Framework. Science. 2013; 341(6145): 514-519. DOI: 10.1126/ science. 1239401.

32. WHO. Atlas of health and climate. Geneva, Switzerland: 2012.

33. Epstein PR. Climate change and emerging infectious diseases. Microbes and Infection. 2001; 3(9): 747754. DOI: 10.1016/S1286-4579(01)01429-0.

34. Ahern MJ, McMichael AJ. Global environmental changes and human health. In: Hester RE, Harrison RM, editors. Global Environmental Change. 17: The Royal Society of Chemistry; 2002. p. 139-60.

35. Ayres J, Forsberg B, Annesi-Maesano I, Dey R, Ebi $\mathrm{K}$, Helms P, et al. Climate change and respiratory disease: European Respiratory Society position statement. Eur Respir J. 2009; 34(2) : 295-302. doi: 10.1183/09031936.00003409.

36. D'Amato G, Cecchi L. Effects of climate change on environmental factors in respiratory allergic diseases. Clin Exp Allergy. 2008; 38(8): 1264-1274. doi: 10.1111/j.1365-2222.2008.03033.x.

37. Patz JA, Vavrus SJ, Uejio CK, McLellan SL. Climate change and waterborne disease risk in the Great Lakes region of the U.S. Am J Prev Med. 2008; 35(5): 451-458. DOI: 10.1016/j. amepre.2008.08.026.

38. Curriero FC, Patz JA, Rose JB, Lele S. The Association between extreme precipitation and waterborne disease outbreaks in the United States, 1948-1994. Am J Public Health. 2001; 91(8): 11941199.

39. McMichael AJ, Woodruff RE, Hales S. Climate change and human health: present and future risks. Lancet. 2006; 367(9513): 859-869.

40. IPCC. Climate change: health impacts and opportunities. 2014.

41. Organización Panamericana de la Salud - OPS. Lineamientos evaluacion de la vulnerabilidad de la salud frente al cambio climático en Colombia. Bogotá: OPS, 2011.

42. Organización Panamericana de la Salud - OPS. Proteger la Salud Frente al Cambio Climatico: evaluación de la vulnerabilidad y la adaptación. Washington: OPS, 2011.

43. Frumkin H, Hess J, Luber G, Malilay J, McGeehin M. Climate Change: The Public Health Response. Am J Public Health. 2008; 98(3): 435-445. DOI: 10.2105/AJPH.2007.119362

44. Kickbusch I, Buckett K. Implementing health in all policies: Adelaide 2010. Adelaide: Department of Health, Government of South Australia. 2010:3-10. 